\title{
Honesty is the best policy
}

$\mathrm{T}$ he series of events as outlined portrays some major concerns about the Institution's IACUC quality control and their check and balance system(s). A deeper investigation of the type of study Katayama was conducting and the overlap that it had with Miller's other protocols is justified for the IACUC. The IACUC should understand how great a deviation this is and the Institutional Official should be informed of the findings, based on potential negative backlash for the institution. The bottom line is that unapproved animal work was done and published in a prestigious journal. An initial step is to notify the journal that the author of record did not have institutional approval or oversight of the study, as was stated in the paper. This could be classified as investigator misconduct, which might result in retraction of the article and potentially negative career implications for Katayama.

The IACUC also has regulatory responsibilities to report this to the appropriate oversight entities. If the work was PHS/NSF-funded, or depending on how the Institutional assurance on file for Great Eastern is written, the IACUC must promptly send a preliminary report to $\mathrm{OLAW}^{1}$ in form of a fax, email or a telephone call. Once full action is determined, a final report should be submitted with actions taken for short and long term corrective plans and a schedule of implementation. If Great Eastern is an AAALAC-accredited institution, they should also notify AAALAC of a potential OLAW investigation ${ }^{2}$. If USDA-covered species were used in this study, there is not a requirement to report until the annual report. But in the spirit of good faith, they may want to make a verbal report to their VMO, to avoid a situation where the VMO hears about the issue in another communication stream (for example, via non-compliance sharing from an intra-agency memorandum of understanding) $)^{3}$. If this is the first incident of this type and it was discovered in a "timely" fashion (this could be very questionable here), and there is immediate correction of the issue and it is reported promptly to Animal Care (either orally or in writing) then the recent introduction of the Animal Care Tech Notes ${ }^{4}$ may be used by Animal Care for this incident.

It is also apparent that Miller's lab was able to use animals from one protocol in another without raising any oversight concerns; this needs to be addressed, and Miller's other studies (as well as any other high-profile studies) should be immediately reviewed to assure there is alignment with approved protocols. It calls into question whether there is an appropriate and functioning Training and Post Approval Monitoring program at the institution, how animals are initially assigned to studies, and if there are mechanisms of assuring correct study assignments. Review and a QC program for assurance are needed.

Since Katayama is no longer at Great Western and the protocol never existed, suspension of this work or this investigator is meaningless. But because this was a collaborative study and Miller was Katayama's mentor, Miller has responsibility for what happened. The Miller lab should be on an elevated status of review and assurance through the IACUC for a defined period of time.

This scenario also begs the ethical question of animal use and how to avoid having to repeat studies, especially if this study is null and void administratively but had scientific merit, as indicated. If the IACUC discovers that Miller and Katayama are truly collaborators, that Miller is using a similar (if not the same) protocol, and that Miller had worked on the study and there had been oversight, then the IACUC has more opportunity to suggest not having to repeat the study, such as assignment of animals and reassignment of authority, given agreement by all. This could also change the nature of some of the notifications to the journal and oversight entities (with full explanations to them). If the work is very disparate and lacked oversight, unfortunately an IACUC-approved study should be repeated.

John J. Hasenau, DVM, DACLAM

Lab Animal Consultants, Sparks, NV, USA.

e-mail:labanimalconsultants@charter.net

Published online: 26 February 2018 https://doi.org/10.1038/s41684-018-0007-x

References

1. Office of Laboratory Animal Welfare. Guidance on Prompt Reporting to OLAW Under the PHS Policy on Humane Care and Use of Laboratory Animals. Notice NOT-OD-05-034. (National Institutes of Health, Washington, DC, 24 February 2005, updated 21 February 2013). https://grants.nih.gov/grants/guide/noticefiles/not-od-05-034.html

2. AAALAC. Accreditation Frequently Asked Questions. https://www. aaalac.org/accreditation/faq_landing.cfm

3. Office of Laboratory Animal Welfare. Memorandum of understanding among the Animal and Plant Health Inspection Service U.S. Department of Agriculture and the Food and Drug Administration U.S. Department of Health and Human Services and the National Institutes of Health U.S. Department of Health and Human Services concerning laboratory animal welfare. (National Institutes of Health, Washington, DC, 24, 1 March 2006). https://grants.nih.gov/grants/olaw/references/finalmou. htm

4 Animal and Plant Health Inspection Service, Animal Care. Tech Notes December 2017 "Incentives for Identifying, Reporting, Correcting, and Preventing Noncompliance with the Animal Welfare Act." http://www.aphis.usda.gov/publications/ animal_welfare/2017/ac-tech-note-incentives-animal-welfare-actcompliance.pdf 